\title{
Editorial
}

\section{Citation errors can be reduced}

The high frequency of citation errors in the medical scientific literature is disturbing. Not only does it indicate carelessness in the preparation of manuscripts and rudeness to previous investigators whose work may never have been read, but it is irritating to readers attempting to trace the referenced studies. Evans et al. evaluated the surgical literature by selecting 50 references from The American Journal of Surgery, Surgery, and Obstetrics, Gynecology and Surgery. Thirteen of the quoted articles could not be traced and, in the remaining 37, they found major errors in 13 and minor errors in $41 . .^{\prime}$ Examining the anaesthetic literature, McLellan $e t$ al. found that half the references that they had selected from Anesthesiology, Anesthesia \& Analgesia, British Journal of Anaesthesia, and Canadian Journal of Anaesthesia contained errors. ${ }^{2}$

Two years ago (June 1992) the CJA editorial board decided that, for all articles accepted for publication, authors would be requested to provide photocopies of the first page of each reference that they quote to accompany their final, revised manuscript. The process was instituted for all articles accepted after January 1st, 1993. On receipt of the final manuscript, the photocopies were checked against the Reference list and modifications were made to the copy-edited version of the manuscript before it was sent to the printers. A final check was introduced in April, 1994 to compare the page proofs with the photocopies. Almost all authors complied with the request and none complained. However, it was anticipated that there might be some delay before it became effective because of the time from provisional acceptance of the article and its eventual publication.

In this issue of the Journal, Asano et al. have determined whether the process has been effective. They compared the frequency of citation errors in 100 selected references from volume 37 (1990) and volume 41 (1994). ${ }^{3}$ They found that the change in policy resulted in an overall decrease of citation errors from 48 to $22 \%$, the number of multiple errors was reduced from 11 to $2 \%$, and the number of major errors from five to three.

From the CJA Editorial Office, Vancouver, B.C.
Simultaneously, a continuing evaluation was also being made "in house" during this period. One hundred citations were chosen from 15 randomly selected articles from each of the six volumes of Canadian Journal of Anaesthesia published from 1989 to 1994 . Major errors, which may prevent or make it difficult to trace citations, were defined as misspelled, incorrect or missing author(s) names, title, journal, year, volume or page number. Minor errors consisted of the use of inappropriate style or punctuation, or failure to identify Editorials or Correspondence in the citation. The results were similar to those of Asano et al. The large error rate $(40-69 \%$ of all citations in 1989-1992) was reduced to $19 \%$ (13 major, 6 minor) in 1993 and to $13 \%$ (11 major, 2 minor) in 1994 (Table). Errors continue to occur but much less frequently. Ongoing documentation of all the alterations to all the references to manuscripts published in 1994 showed that an average of 17 modifications was made to each article.

We believe that citation accuracy is important. Scientific progress is made only by improving on the past. If references to previous work cannot be found or, worse, the work is quoted but never read ("perpetual errors" - citations reproduced faithfully from another article along with existing errors), science is ill-served. Authors and readers are frustrated if they are unable to trace erroneous citations and this is particularly important for references from unusual or historical sources. It is hoped that by asking authors to provide supporting evidence for their citations, more might be read. Editors, publishers, and authors share the responsibility of providing accurate manuscripts. ${ }^{4}$

The elimination of citation errors may be impossible but Asano et al. have confirmed that the frequency can be reduced considerably. However, to do so, involves time and labour and would, no doubt, be well suited to computerized checking systems. ${ }^{5}$ Our experience with Medline during this process has been encouraging. Although errors also occur in its data base, all that we have identified have been minor and did not prevent retrieval of source material. There is no point, but much frustration, in quoting a reference in which the citation is inaccurate or fictitious. 
TABLE Errors per 100 references analyzed in Canadian Journal Anaesthesia, 1989-1994

\begin{tabular}{lllllll}
\hline & 1989 & 1990 & 1991 & 1992 & 1993 & 1994 \\
\hline Major & 32 & 44 & 28 & 33 & 13 & 11 \\
Minor & $\frac{37}{69}$ & $\frac{15}{59}$ & $\frac{12}{40}$ & $\frac{30}{63}$ & $\frac{6}{19}$ & $\frac{2}{13}$ \\
Total & 69 & & & &
\end{tabular}

We would like to thank all authors who have published their work in the Canadian Journal of Anaesthesia during the last two years for their cooperation and support.

\section{Le nombre des références erronées peut être réduit}

Il faut s'inquiéter du grand nombre d'erreurs de références bibliographiques relevées dans nos revues médicales. Non seulement elles témoignent de la négligence de celui qui soumet un manuscrit pour publication mais aussi de son impolitesse impardonnable envers les auteurs qu'il cite et dont le travail pourrait ne jamais être consulté; de plus, ces erreurs exaspèrent tous ceux qui essayent de retracer les références concernant des études sur lesquelles elles s'appuyent. En chirurgie, Evans et al. ont sélectionné 50 références tirées de l'American Journal of Surgery, de Surgery et d'Obstetrics, Gynecology and Surgery. Treize des articles cités n'ont pu être retracés, et parmi les 37 autres, 13 comportaient des erreurs majeures et 41 des erreurs mineures. ${ }^{1}$ Dans un relevé de la littérature pertinente à anesthésie, McLellan et al. ont trouvé que la moitié des références choisies dans Anesthesiology, Anesthesia \& Analgesia, The British Journal of Anaesthesia et le Journal canadien d'anesthésie contenaient des erreurs. ${ }^{2}$

Il y a deux ans (en juin 1992), le conseil de rédaction du Journal canadien d'anesthésie décidait de demander aux auteurs dont les articles étaient acceptés pour publication de fournir pour l'édition finale de leur manuscrit une photocopie de la première page de chacune des références de leur bibliographie. Ce mécanisme fut instauré pour tous les articles acceptés pour publication après le premier janvier 1993. Sur réception de la version finale du manuscrit, les photocopies ont été comparées avec la bibliographie originale et des modifications ont été apportées à la copie révisée avant son expédition aux imprimeurs. Une vérification ultime a été initiée en avril 1994 dans le but de comparer les épreuves dimprimerie aux photocopies. La plupart des auteurs se sont pliés à cette exigence et aucun ne s'en est plaint. Cependant, nous avons prévu que cette façon de procéder ajouterait un délai supplémentaire au temps écoulé entre l'acceptation provisoire et la publication eventuelle.

Dans ce numéro du Journal, Asano et al. évaluent l'efficacité du processus. Ils comparent la fréquence des erreurs trouvées dans 100 références bibliographiques choisies dans le volume 37 (1990) et le volume 41 (1994). ${ }^{3}$ Ils rapportent que ce changement de politique a eu pour résultat de réduire le nombre total des erreurs de $48 \%$ à $22 \%$, celui des erreurs multiples de $11 \%$ à $2 \%$ et le nombre des erreurs majeures de cinq à trois.

Simultanément, pendant la même période, l'évaluation en interne s'est continuée. Cent références ont été choisies dans 15 articles désignés au hasard dans chacun des six volumes du Journal canadien d'anesthésie publiés de 1989 à 1994. Etaient définies comme erreurs majeures, c.-àd., celles qui pouvaient empêcher ou gêner la recherche d'une référence: le noms des auteurs, le titre, le journal, l'année, le volume et le numéro de page mal orthographiés, incorrects ou absents. Les erreurs étaient considérés comme mineures lorsque la ponctuation ou le style était inapproprié, ou lorsqu'on ne spécifiait pas s'il s'agissait d'un éditorial ou d'une lettre à la rédaction. Les résultats ont été identiques à ceux d'Asano et al. Le pourcentage élevé d'erreurs (40-69\% de toutes les références de 1989 à 1992) avait baissé à $19 \%$ (13 majeures, 6 mineures) en 1993, et à $13 \%$ (11 majeures et 2 mineures) en 1994 (Tableau). Des erreurs surviennent toujours mais moins souvent. Le compte rendu au jour le jour de toutes les corrections apportées aux références provenant des manuscrits publiés en 1994 a montré que 17 modifications en moyenne avaient été effectuées sur chacun des articles.

Nous croyons qu'il est important que les références soient précises. La science ne peut progresser qu'en améliorant le passé. Si des citations se référant à des travaux antérieurs ne peuvent être retrouvées, ou, ce qui est pire, qu'un ouvrage soit cité sans n'être jamais consulté (" erreurs sempiternelles" - références comportant des erreurs pré-existantes répétées d'un article à un autre), la science sera mal servie. Les auteurs comme les lecteurs se sentent frustrés s'ils ne pas réussissent à retracer les citations particulièrement lorsque leur origine est unusuelle ou historique. Nous espérons qu'en demandant aux auteurs de fournir l'evidence qui soutient leurs références, celles-ci seront consultées plus souvent. Les éditeurs, les rédacteurs et les auteurs sont conjointement responsables de la précision du manuscrit. ${ }^{4}$

L'élimination des erreurs de citation peut s'avérer impossible mais Asano et al. confirment que leur fréquence 
TABLEAU Nombres d'erreurs par 100 références analysee dans le Journal canadien d'anesthésie, 1989-1994

\begin{tabular}{lllllll}
\hline & 1989 & 1990 & 1991 & 1992 & 1993 & 1994 \\
\hline Majeures & 32 & 44 & 28 & 33 & 13 & 11 \\
Mineures & $\frac{37}{69}$ & $\frac{15}{59}$ & $\frac{12}{40}$ & $\frac{30}{63}$ & $\frac{6}{19}$ & $\frac{2}{13}$ \\
Total & 69 & 59 &
\end{tabular}

peut être réduite au minimum. Cependant, ceci nécessite du temps et du travail; un système informatisé de vérification peut être utile. ${ }^{5}$ Notre expérience avec Medline est encourageante. Bien que des erreurs puissent se produire dans la base de données, toutes celles que nous avons identifiées étaient mineures et n'empêchaient pas de retrouver la source citée. Il est vain et, de plus, frustrant de citer une référence inexacte ou fictive.

Nous désirons remercier tous les auteurs qui ont publié au cours des deux dernières années un travail dans le Journal canadien d'anesthésie, pour leur collaboration et leur appui.

\section{References}

1 Evans JT, Nadjari HI, Burchell SA. Quotational and reference accuracy in surgical journals: a continuing peer review problem. JAMA 1990; 262: 1353-4.

2 McLellan $M F$, Case $L D$, Barnett $M C$. Trust, but verify: the accuracy of references in four anesthesia journals. Anesthesiology 1992; 77: 185-8.

3 Asano M, Mikawa $K$, Nishina $K$, Maekawa $N$, Obara $H$. Improvement of the accuracy of references in the Canadian Journal of Anaesthesia. Can J Anaesth 1995; 41: 370-2.

4 Biebuyck JF. Concerning the ethics and accuracy of scientific citations (Editorial). Anesthesiology 1992; 77: 1-2.

5 Rosman AS, Schachner JR. A better citation system (Letter). Anesthesiology 1993; 78: 614 . 